\title{
T2DM-GeneMiner a web resource for meta-analysis and marker identification for type 2 diabetes mellitus Axel Rasche* and Ralf Herwig
}

\author{
Address: Department Vertebrate Genomics, Max Planck Institute for Molecular Genetics, Ihnestr. 63-73, D-14195 Berlin, Germany \\ Email: Axel Rasche* - rasche@molgen.mpg.de \\ * Corresponding author
}

from Third International Society for Computational Biology (ISCB) Student Council Symposium at the Fifteenth Annual International Conference on Intelligent Systems for Molecular Biology (ISMB)

Vienna, Austria. 2I July 2007

Published: 20 November 2007

BMC Bioinformatics 2007, 8(Suppl 8):P3 doi:10.1 186/147|-2105-8-S8-P3

This abstract is available from: http://www.biomedcentral.com/I47I-2105/8/S8/P3

(c) 2007 Rasche and Herwig; licensee BioMed Central Ltd.

\section{Background}

Multiple functional genomics data for complex human diseases have been published and made available by researchers worldwide. Main goal of these studies is the detailed analysis of a particular aspect of the disease. Recently, meta-analysis approaches have been published that try to extract meaningful disease genes and networks by integrating and combining these individual studies using bioinformatics strategies.

\section{Results}

Here we report on a meta-analysis approach that combines high-throughput data of heterogeneous origin in the domain of type 2 diabetes mellitus (T2DM), in particular in connection with obesity as a risk factor. Different data sources such as DNA microarrays, ChIP on chip and qualitative data from multiple tissues from human and mouse are integrated and validated by a scoring system in order to assign disease relevance to the genes. Using a random sampling approach we computed a set of 213 genes most relevant for obesity-induced T2DM. Furthermore, we extrapolated functional information on cellular networks associated with these genes such as pathway information, protein-protein interactions and gene regulatory networks. In order to allow users to derive T2DM relevance for any given gene we have set up a web interface that allows the screening of the gene in the light of the underlying data (T2DM-GeneMiner http://t2dm-genem iner.molgen.mpg.de).

\section{Conclusion}

Using a simple scoring algorithm we computed a core set of 213 genes that show significant disease relevance in the data sets under study. These genes have been further validated in the functional context of networks and exhibit high potential for understanding diabetic pathways and pathway cross-talk. Our web resource allows the user to access the information that was gathered and to assess disease relevance for any human or mouse gene. Thus, we conclude that our study is a valuable resource for diabetes research and a template for meta-analysis studies in other disease domains. 